\title{
ARTIGOS
}

Submetido 23.05.2017. Aprovado 09.04.2018

Este artigo é de coautoria de um membro do Corpo Editorial Científico da RAE e foi avaliado pelo processo de double blind review com imparcialidade e independência. Editor Científico: Delane Botelho

Versão original

DOI: http://dx.doi.org/10.1590/So034-759020180605

\section{A IMPORTÂNCIA DO APEGO À MARCA PARA O ENGAJAMENTO EM CAUSAS DE RESPONSABILIDADE SOCIAL CORPORATIVA}

\author{
Brand attachment importance to corporate social responsibility causes \\ adherence
}
La importancia del apego de marca para la adhesión a causas de responsabilidad social corporativa

\begin{abstract}
RESUMO
Este estudo examina o efeito conjunto da congruência marca-causa, do apego à marca e da atitude em relação à causa de Responsabilidade Social Corporativa (RSC) sobre a intenção dos consumidores de se engajarem em causas de RSC patrocinadas por uma marca. Os resultados de dois experimentos sugerem que, quando a atitude do consumidor em relação à causa é menos positiva e o apego do consumidor à marca é baixo, a intenção de aderir à causa de RSC é maior quando a causa é congruente com a marca do que quando a causa é incongruente com a marca; se o apego do consumidor é alto, a intenção de aderir à causa não depende da congruência marca-causa. Por outro lado, se a atitude do consumidor em relação à causa é mais positiva, o apego à marca não modera o efeito da congruência sobre a intenção de aderir à causa. A percepção de intenção persuasiva mediou a influência da congruência marca-causa sobre a intenção de aderir para pessoas de baixo apego à marca, mas não para pessoas de alto apego à marca.
\end{abstract}

PALAVRAS-CHAVE | Responsabilidade social corporativa, apego à marca, atitude em relação à causa, congruência marca-causa, experimento.

\section{KAMIYA'}

annaysamuniz@gmail.com

ORCID: 0000-0002-5524-709X

\section{ABSTRACT}

This study examines the joint effect of brand-cause congruence, brand attachment, and attitude toward corporate social responsibility (CSR) on the intention of consumers to adhere to causes sponsored by a brand. The results of two experiments suggest that when consumer attitude toward such causes is less positive and attachment to the brand is low, the intention to adhere to a CSR cause is greater when the brand is congruent to the cause than when it is incongruent; if consumer brand attachment is high, the intention to adhere to the cause is the same, regardless of brand-cause congruence. On the other hand, if consumer attitude toward the cause is more positive, brand attachment does not moderate the effect of brand-cause congruence on the intention to adhere to the cause. The perception of persuasive intent mediates the influence of brand-cause congruence on the intention to adhere for consumers with low brand attachment, but not for those with high brand attachment. KEYWORDS / Corporate social responsibility, brand attachment, attitude toward the cause, brand-cause congruence, experiment.

karoline.agnne@gmail.com

ORCID: 0000-0002-0057-2805

\section{DÉBORA BESERRA RAMOS}

deboraramoscontatos@gmail.com

ORCID: 0000-0002-7015-4780

\section{${ }^{1}$ Centro Universitario da}

FEl, Departamento de Pós-

Graduação, São Paulo, SP, Brasil

${ }^{2}$ Universidade de São Paulo, Escola de Artes, Ciências e Humanidades, Departamento de Marketing, São Paulo, SP, Brasi

\section{RESUMEN}

Este estudio analiza el efecto combinado de la congruencia marca-causa, del apego a la marca y de la actitud hacia la causa de Responsabilidad Social Corporativa (RSC) sobre la intención de los consumidores de adherir a causas de RSC patrocinadas por una marca. Dos experimentos sugieren que cuando la actitud del consumidor hacia la causa es menos positiva y el apego es bajo, la intención de adherir a la causa es más alta cuando esta es congruente con la marca que cuando es incongruente. Si el apego es alto, la intención de adherir no depende de la congruencia marca-causa. Por otro lado, si la actitud del consumidor hacia la causa es más positiva, el apego no modera el efecto de la congruencia marca-causa sobre la intención de adherir. En el caso de las personas con bajo apego, la percepción del intento persuasivo medió la influencia de la congruencia marca-causa sobre la intención de adherir. Esto no se observó entre las personas con alto apego.

PALABRAS CLAVE I Responsabilidad social corporativa, apego a la marca, actitud en relación a la causa, congruencia marca-causa, experimento. 


\section{INTRODUÇÃO}

Questões como desigualdade social, violência urbana, desastres ambientais e deficit educacional têm ocupado cada vez mais espaço no debate nacional, estimulando as empresas a assumirem papéis mais relevantes na solução desses problemas. As ações de responsabilidade social contribuem não apenas para as empresas (Mohr, Webb, \& Harris, 2001), mas também para as pessoas que se beneficiam das ações sociais. 0 patrocínio de ações de RSC tem efeitos positivos sobre a intenção de compra (Andrews, Luo, Fang, \& Aspara, 2014; Sen \& Bhattacharya, 2001), a intenção de investir na empresa (Sen, Bhattacharya, \& Korschun, 2006), a atitude em relação à empresa (Zdravkovic, Magnusson, \& Stanley, 2010) e o boca a boca positivo (Skarmeas \& Leonidou, 2013).

Embora os benefícios das ações de Responsabilidade Social Corporativa (RSC) para as empresas já estejam bem documentados, o mesmo não se pode dizer sobre a intenção dos consumidores em também contribuir para a causa. A identificação do consumidor com a causa patrocinada por uma marca pode aumentar a sua vontade de também participar, alavancando os efeitos positivos tanto para as pessoas beneficiadas pela causa quanto para a própria marca.

Apesar dos seus aspectos positivos, as ações de RSC também podem ter efeitos negativos para a marca e para o engajamento do consumidor (Becker-Olsen, Cudmore, \& Hill, 2006). De acordo com Vlachos, Tsamakos, Vrechopoulos e Avramidis (2009), os consumidores podem não perceber a reciprocidade das ações de RSC, atribuindo motivações oportunistas ao engajamento das empresas em tais práticas, além de percebê-las como tentativas deliberadas de enganá-los sobre a capacidade ética da empresa (Skarmeas \& Leonidou, 2013).

A congruência entre a marca e a ação de RSC, grau em que uma marca e uma causa social compartilham os mesmos valores ou a mesma base de consumidores (Sen \& Bhattarcharya, 2001), é fator essencial para que uma marca possa obter resultados positivos com o patrocínio de causas sociais (Roy, 2010; Zdravkovic et al., 2010) Entretanto, outros estudos (Nan \& Heo, 2007; Sheikh \& Beise-Zee, 2011) sugerem que o efeito negativo da baixa congruência marca-causa possa ser mitigado. Uma pergunta que ainda não foi adequadamente respondida é se uma forte relação afetiva entre o consumidor e a marca patrocinadora de uma causa social ou uma atitude muito positiva do consumidor em relação à causa social poderiam diminuir o impacto negativo da baixa congruência marca-causa.

He, Zhu, Gouran e Kolo (2016) mostraram que o apego, medida do vínculo emocional que um consumidor possui em relação à uma marca, influencia positivamente a intenção de compra de uma marca patrocinadora de causas sociais. 0 apego à marca, além de importante sinalizador da identificação com a marca, também promove a lealdade e o comprometimento dos consumidores (Hazan \& Shaver, 1994). Entretanto, o efeito do apego em relação à marca em condições de baixa congruência marca-causa ainda não foi examinado empiricamente.

Por outro lado, Barone, Norman e Miyazaki (2007) sugerem que a atitude mais positiva em relação à causa social pode mitigar o efeito negativo da baixa congruência marca-causa. 0 que não está claro, entretanto, é se uma atitude positiva em relação à marca poderia mitigar o efeito de níveis baixos de apego à causa em condições de baixa congruência marca-causa.

Diversos estudos sugeriram que a baixa congruência marcacausa levaria os consumidores a suspeitar da motivação da marca, mas poucos estudos examinaram os mecanismos pelos quais a congruência marca-causa influencia as respostas ao patrocínio de uma causa social (entretanto, veja exceções como Romani \& Grappi, 2014; Romani, Grappi, \& Bagozzi, 2014). Neste estudo, propomos que a percepção de intenção manipulativa, grau em que o consumidor percebe que uma campanha de propaganda esteja tentando persuadi-lo de maneira injusta (Campbell, 1995), é responsável por mitigar os efeitos positivos do patrocínio de uma causa social. Essa proposta é coerente com o modelo de conhecimento da persuasão (Friestad \& Wright, 1994) segundo o qual os consumidores desenvolvem conhecimento sobre as táticas persuasivas usadas pelas empresas, aumentando o ceticismo em relação às propagandas (Obermiller \& Spangenberg, 1998) e influenciando negativamente as suas respostas comportamentais.

Este estudo contribui, portanto, para a literatura de RSC ao examinar conjuntamente três variáveis que determinam os resultados do patrocínio de uma ação de RSC - a congruência marca-causa, o apego à marca e a atitude em relação à causa de RSC. Embora essas variáveis tenham sido examinadas isoladamente em estudos anteriores, o exame conjunto de variáveis importantes contribui para ampliar o conhecimento sobre um determinado fenômeno de interesse, como o patrocínio de ações de RSC. 0 estudo também contribui para a literatura ao examinar o efeito do patrocínio de ações de RSC sobre uma variável de interesse social, a intenção de aderir à causa. Finalmente, o presente estudo contribui para a teoria de RSC ao examinar um dos mecanismos pelos quais a congruência marcacausa influencia os resultados do patrocínio de ações de RSC.

Do ponto de vista gerencial, este estudo sugere às empresas as condições em que a congruência marca-causa é mais ou menos importante, servindo como guia na seleção das causas que as marcas devem apoiar e os resultados que podem ser esperados. 
Nas próximas seções, discutimos os conceitos envolvidos na formulação das hipóteses e apresentamos os dois estudos que testaram essas hipóteses. Concluímos com as implicações teóricas e gerenciais e apontamos as limitações do estudo, bem como as sugestões de estudos futuros.

\section{RESPONSABILIDADE SOCIAL CORPORATIVA}

Mohr et al. (2001) definem a RSC como "o compromisso da empresa em minimizar ou eliminar quaisquer efeitos prejudiciais e maximizar seu impacto benéfico a longo prazo" (p. 47). 0 engajamento das empresas nesse tipo de iniciativa é atraente, pois está positivamente relacionado à performance financeira das empresas (Stanwick \& Stanwick, 1998), à atitude e à intenção de compra dos consumidores (Andrews et al., 2014; Mohr et al., 2001) e à intenção de investir na empresa (Sen et al., 2006).

Sen e Bhattacharya (2001) sugeriram que os indivíduos tendem a se identificar com marcas que se engajam em RSC, pois isso auxiliaria a manutenção da autoimagem positiva do indivíduo. Contudo, esses mesmos autores identificaram também que a relação entre o patrocínio de ações de RSC e as respostas comportamentais do indivíduo podem variar de acordo com o apoio pessoal em relação às questões de RSC, às suas crenças e à congruência percebida entre as suas próprias características e as da marca. Além disso, quando a causa de RSC tem alta congruência com a marca, as atitudes do consumidor em relação à marca são mais favoráveis (Nan \& Heo, 2007; Roy, 2010). Uma marca pode ter maior congruência com a causa se ambas servem uma base de consumidores semelhantes ou se possuem valores semelhantes.

A maioria dos estudos sobre a RSC, entretanto, tem se concentrado mais nos retornos dessas iniciativas para os negócios da empresa e muito menos em seus possíveis retornos sociais (Bhattacharya \& Sen, 2004). Por exemplo, Romani e Grappi (2014) verificaram que testemunhar a promoção de boas ações de uma empresa pode aumentar a probabilidade de que os indivíduos se envolvam em comportamentos sustentáveis semelhantes.

Em sua proposta de um quadro referencial para a integração adequada das ações de RSC às atividades de marketing das organizações, Maignan e Ferrell (2004) sugerem que muitos estudos não consideraram adequadamente como as práticas e o pensamento de marketing podem contribuir para o desenvolvimento de práticas socialmente responsáveis dentro das organizações. Maignan e seu colega também criticam os estudos na área de marketing que examinaram as respostas dos consumidores às ações de RSC usando dimensões limitadas e medidas de resposta relacionadas exclusivamente ao desempenho de marketing. No presente estudo, além de considerar a resposta dos consumidores às diferentes dimensões de RSC, também examinamos uma medida de resposta raramente investigada na literatura, que é a intenção do consumidor em se engajar na causa defendida pela marca.

\section{APEGO À MARCA}

0 interesse sobre o estudo do apego à marca surgiu com o reconhecimento da importância da criação de relações fortes entre consumidores e marcas. 0 apego influencia a construção de relacionamentos fortes com a marca, aumentando a lealdade dos consumidores e tornando-os menos sensíveis a preços (Park, MacInnis, \& Priester, 2008). Apego à marca pode ser definido como "a força da relação cognitiva e afetiva que conecta uma marca com o indivíduo [...] e denota um estado psicológico em que a marca é vista como uma extensão do eu" (Park et al., 2008, p. 4).

0 apego à marca é um importante moderador das respostas dos indivíduos às ações de RSC, porque elevados níveis de apego tornam o consumidor mais sensível e menos crítico às atividades de uma marca (Park, MacInnis, Priester, Eisingerich, \& lacobucci, 2010). Segundo He et al. (2016), o apego emocional à marca está positivamente relacionado à intenção do indivíduo de comprar produtos da marca patrocinadora de uma campanha de RSC, devido à imagem socialmente responsável da marca, além de fortalecer a identidade moral do indivíduo. Como veremos na próxima seção, o apego à marca pode levar um consumidor a demonstrar respostas positivas a despeito de uma baixa congruência marca-causa.

\section{O EFEITO DO APEGO À MARCA SOBRE A CONGRUÊNCIA MARCA-CAUSA DE RSC}

Roy (2010) observou que o patrocínio de ações de RSC incongruentes com a marca é percebido de maneira negativa e, quando a congruência é alta, a percepção de sinceridade e a atitude em relação à marca são mais positivas. Da mesma forma, Becker-Olsen et al. (2006) observaram que a baixa congruência marca-causa pode influenciar negativamente as crenças, atitudes e intenções dos consumidores, independentemente da motivação da empresa patrocinadora. 
Apesar dos resultados desses estudos, ainda há dúvidas sobre os resultados que podem ser obtidos em situações de baixa congruência e alto apego, uma vez que há evidências teóricas de que a consciência do consumidor sobre a causa, a credibilidade da empresa, a atitude em relação à causa ou o conteúdo da comunicação podem mitigar o efeito da baixa congruência (Barone et al., 2007; Nan \& Heo, 2007).

Em relação aos efeitos "secundários" das ações de RSC, Kwak e Kwon (2016) sugeriram que a identificação do consumidor com uma empresa aumenta a percepção de que ele próprio está envolvido no processo de doação, evocando sentimentos de gratidão e encorajando-o a colaborar com tais iniciativas. Quanto maior a identificação consumidor-empresa, maiores o sentimento de gratidão e a intenção de apoiar as ações. Os resultados de Sen, Johnson, Bhattacharya e Wang (2015) também dão indícios de que níveis elevados de apego à marca podem estar positivamente relacionados à intenção dos indivíduos de aderir a ações de RSC, independentemente da congruência marca-causa.

Um consumidor com elevados níveis de apego à marca pode demonstrar um processo de assimilação enviesado, tendência de assimilar os eventos que concordam com o próprio ponto de vista como mais confiáveis (Lord, Ross, \& Lepper, 1979). Na prática, esse viés assimilativo levaria os consumidores a esquecerem os "erros" cometidos pelas marcas (McCullought et al., 1998) e engajarem-se em processamento enviesado e defensivo de informações negativas relacionadas à marca (Schmalz \& Orth, 2012).

Propomos que níveis elevados de apego à marca podem moderar o efeito da congruência marca-causa sobre as respostas dos indivíduos. Especificamente, um nível elevado de apego à marca pode levar um indivíduo a descontar uma baixa congruência marca-causa, inibindo a sua desconfiança da motivação da marca mesmo que ela decida patrocinar causas com baixa congruência. Por outro lado, indivíduos com baixo apego à marca só responderão positivamente quando a marca for congruente com a causa patrocinada. Em outras palavras, propomos que níveis elevados de apego à marca podem mitigar o efeito negativo da incongruência marca-causa, conduzindo às seguintes hipóteses:

H1a: A intenção de aderir a uma ação de RSC promovida por uma marca é maior quando a causa é congruente com a marca do que quando a causa é incongruente para consumidores que têm baixo apego à marca.

H1b: A intenção de aderir a uma ação de RSC promovida por uma marca independe da congruência marca-causa para consumidores que têm alto apego à marca.

\section{O PAPEL MEDIADOR DA INTENÇÃO PERSUASIVA}

Os indivíduos são constantemente expostos a situações em que têm de lidar com mensagens e ações de marketing que usam diferentes apelos para convencê-los a comprar ou usar produtos e serviços. A exposição a esses apelos estimula o desenvolvimento de um conhecimento sobre as tentativas de persuasão (Friestad \& Wright, 1994) e é usada pelos indivíduos para interpretar e avaliar tais mensagens.

Obermiller e Spangenberg (1998) sugeriram que o conhecimento sobre as táticas de persuasão aumenta o ceticismo em relação às propagandas, que, por sua vez, reduz a probabilidade de respostas positivas às causas associadas à empresa (Lawler \& Thye, 2006). Romani et al. (2014) sugeriram que persuadir os consumidores de que as ações de RSC da empresa são desinteressadas e altruístas é essencial para produzir respostas positivas, uma vez que a percepção de razões oportunistas pode motivar os consumidores a duvidarem dos esforços da marca. Skarmeas e Leonidou (2013) observaram que os consumidores se tornam mais resistentes a informações positivas e mais propensos ao boca a boca negativo quando duvidam dos motivos da marca em se engajar em ações de RSC.

Mohr et al. (2001) identificaram que os indivíduos acreditam que parte da motivação das empresas para agirem de maneira socialmente responsável é o interesse em ajudar, mas que as empresas também têm interesses próprios. Chiou, Hsu e Hsieh (2013) investigaram a relação entre o apego à marca e a percepção de intenção persuasiva e descobriram que, para elevados níveis de apego, há uma tendência à negação de informações negativas atribuídas à marca, já que os indivíduos são menos propensos a se influenciarem negativamente por suspeitas ou acusações que não se alinham com suas crenças em relação à marca.

Assim, propomos que pessoas com alto apego à marca são menos sensiveis à percepção de intenção persuasiva das ações de RSC, independentemente da congruência marca-causa. Por sua vez, pessoas com baixo apego à marca são mais sensíveis à intenção persuasiva da marca e podem perceber, em ações de baixa congruência, uma intenção manipulativa. Formalmente, propomos, então, a seguinte hipótese:

H2: A percepção de intenção persuasiva mediará o efeito da congruência marca-causa sobre a intenção de aderir à causa para pessoas com baixo nível de apego à marca, mas não para pessoas com alto nível de apego à marca. 


\section{EXPERIMENTO 1}

Neste experimento, investigamos o efeito moderador do apego à marca sobre a relação entre a congruência marca-causa e a intenção do indivíduo em aderir a uma ação de RSC ( $\mathrm{H}_{1}$ a e $\left.\mathrm{H}_{1} b\right)$ e o efeito mediador da intenção persuasiva para pessoas com baixo nível de apego, mas não para pessoas com alto nível de apego $\left(\mathrm{H}_{2}\right)$.

\section{Método}

\section{Amostra e design}

Estudantes universitários ( $n=131,59 \%$ homens, $M=21$ anos) foram convidados a participar de um experimento de laboratório com duas condições (congruência marca-causa: congruente vs. incongruente), entre sujeitos, em que o apego à marca foi mensurado. Os participantes foram abordados durante o intervalo das aulas e convidados a responder a uma pesquisa em um laboratório. Ao acessarem o questionário, o programa de computador fazia a alocação aleatória das condições experimentais aos participantes.

\section{Pré-teste}

Fizemos um pré-teste para selecionar uma marca e duas ações de RSC, uma congruente e outra incongruente com a marca. Os participantes do pré-teste $(n=31)$ pertenciam à mesma população dos participantes do estudo principal. Inicialmente, cada indivíduo foi exposto a 11 opções de ações de RSC e solicitado a indicar as duas ações de RSC com as quais ele mais se identificava e, em seguida, foi solicitado a indicar a marca que ele considerava mais apropriada para executar as 11 ações de RSC. As 37 marcas apresentadas foram selecionadas com base no julgamento dos pesquisadores sobre a congruência entre a marca e as causas investigadas.

A ação de reciclagem coletiva foi a mais citada (15 citações ou $48 \%$ dos respondentes), sendo escolhida como estímulo do experimento. A marca mais associada à reciclagem coletiva foi a Coca-Cola, citada por 21 dos 31 respondentes, sendo também escolhida como estímulo. Com base em nosso julgamento, selecionamos uma ação de RSC que fosse incongruente com a marca Coca-Cola (prevenção do câncer de pele).

\section{Procedimentos}

No início do experimento, os participantes foram informados de que a Coca-Cola estava testando uma nova propaganda de uma ação de RSC antes de um amplo lançamento nacional. Após a exposição a uma das propagandas, os participantes indicaram sua intenção em aderir à campanha $(a=.78 ; M=4,57 ; D P=$ $1,20)$, concordando ou discordando ( 1 = discordo totalmente e 7 = concordo totalmente) com as três afirmações adaptadas de Romani et al. (2014) ("Esta propaganda influenciou positivamente minha opinião sobre a reciclagem coletiva”, "Minha disposição a colaborar/aderir com a reciclagem coletiva aumentou depois de ver esta propaganda" e "Esta propaganda me incentivou a buscar informações sobre a reciclagem coletiva”).

A percepção de intenção persuasiva foi medida utilizando a escala de Inferências de Intenção Manipulativa (IMI) ( $a=.82 ; M$ $=2,23 ; \mathrm{DP}=1,00$ ) de Campbell (1995). Para medir a congruência marca-causa, adaptamos a escala usada por Keller e Aaker (1992) e Smith e Park (1992) ( $a=.90 ; M=4,44 ; D P=1,77)$, usando escalas do tipo Likert com 7 pontos.

Por fim, os participantes indicaram em que medida a marca despertava um conjunto de 10 sentimentos (alegre, feliz, satisfeito, relaxado, inspirado, motivado, interessado, ativo, entusiasmado e tranquilo) usando uma escala de 7 pontos ( 1 = nada; 7 = muito). Esses itens foram adaptados de Thomson, Maclnnis e Park (2005) e foram agrupados em um índice $(a=.93 ; M=4,03 ; D P=1,47)$ para representar o apego à marca. Depois de completar essas medidas, os participantes responderam a questões demográficas e receberam um agradecimento pela participação.

\section{Resultados}

\section{Checagem da manipulação}

Como esperado, a congruência percebida entre a marca CocaCola e a ação de RSC “Reciclagem coletiva" $(M=5,6)$ foi maior do que a congruência percebida entre Coca-Cola e a ação de RSC "Proteção contra o câncer de pele" $(M=3,2$; $t(129)=10,7$; $p<0,01)$, significando que a manipulação da congruência marcacausa funcionou como previsto.

\section{Intenção de aderir à campanha}

A análise do efeito da congruência marca-causa sobre a intenção de aderir à ação de RSC moderado pelo apego à marca ( $\mathrm{H}_{1}$ a e $\mathrm{H} 1 \mathrm{~b}$ ) foi feita com o uso da macro PROCESS (Hayes, 2013; Modelo 1) usando 10 mil reamostras pelo sistema de bootstrapping. 0 procedimento foi realizado utilizando a variável intenção de aderir como dependente, o apego à marca como moderadora e a congruência marca-causa como variável independente (o = incongruente; 1 = congruente). 
Os resultados mostraram que a regressão é significativa $\left(F(3,127)=13,53, p<0,01 ; R^{2}=0,24\right)$. Para a amostra de 131 respondentes, o cálculo post-hoc do poder do teste do teste $t$ do coeficiente de interação da regressão, calculado pelo software G-Power (Faul, Erdfelder, Lang, \& Buchner, 2007), superou 99,9\%. Confirmando a hipótese de moderação, o coeficiente do termo da interação foi significativo ( $b=-0,38$; $t$ (127) = -2,98, $p<0,01)$. Para decompor essa interação, utilizamos a técnica de Johnson-Neyman para identificar o intervalo de apego à marca para o qual o efeito da congruência da campanha foi significativo (Spiller, Fitzsimons, Lynch, \& McClelland, 2013). Quando o apego à marca assumiu valores inferiores a 4,21, correspondentes a 49\% da amostra, houve um efeito significativo e positivo $(b=0,37, D P=0,19, p=0,05)$ da congruência sobre a intenção de aderir à ação de RSC. Confirmando $\mathrm{H}_{1 \mathrm{a}}$ e $\mathrm{H}_{1 \mathrm{~b}}$, quando o nível de apego era baixo (valores inferiores a 4,21), a intenção de adesão à campanha de RSC foi maior para a campanha congruente do que para a campanha incongruente. Para níveis altos de apego à marca (maior que 4,21), não verificamos diferença significativa da intenção de adesão de acordo com a congruência marca-causa (Gráfico 1).

\section{Gráfico 1. Intenção de aderir à causa por congruência e por nível de apego à marca}

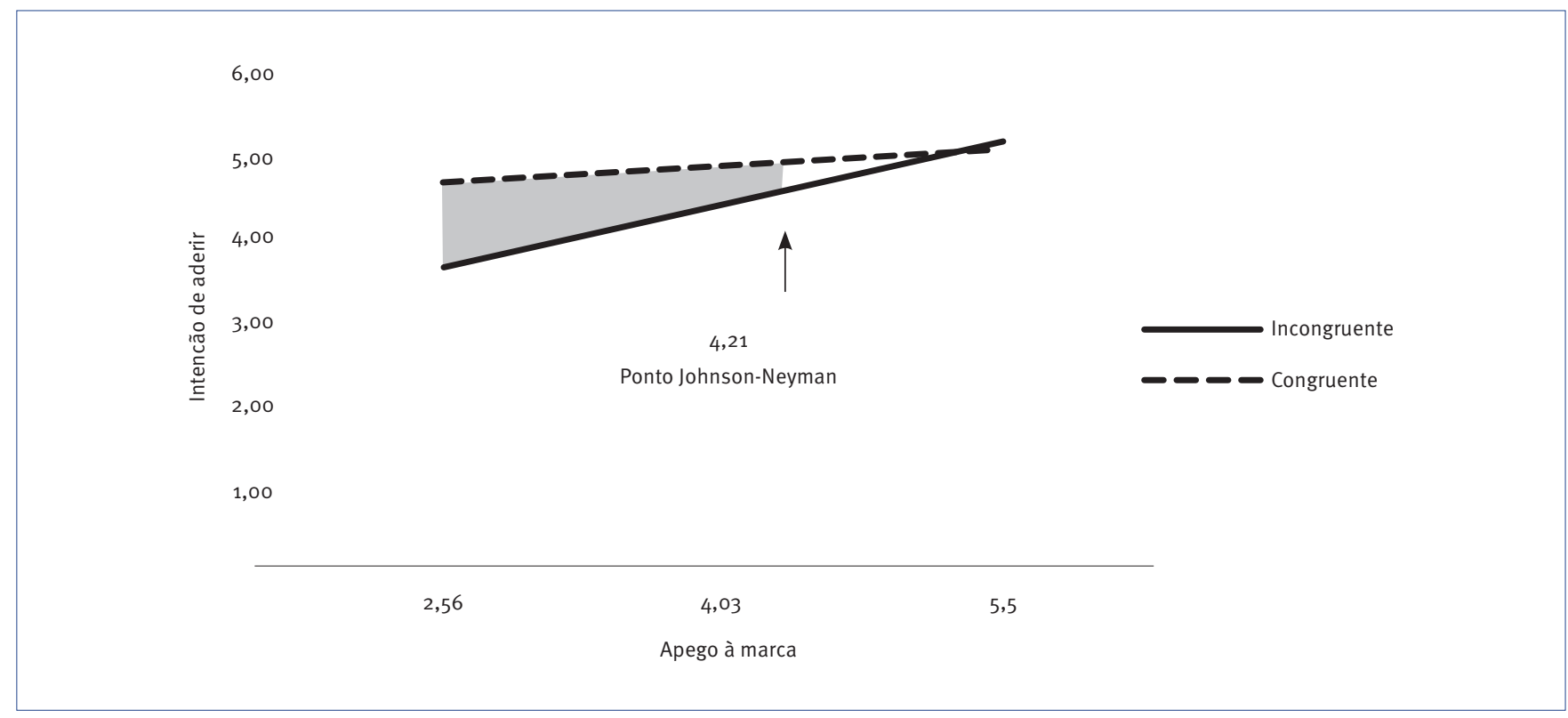

\section{O efeito mediador da intenção persuasiva}

Em seguida, fizemos uma análise de mediação moderada para testar $\mathrm{H}_{2}$. Usamos o modelo 8 da macro PROCESS (Hayes, 2013) considerando intervalos de confiança de $95 \%$ gerados com 10 mil reamostras. Como previmos, verificamos um efeito condicional indireto significativo e positivo da intenção persuasiva ( $b=2.56[.03 ; .51])$ para baixos níveis de apego em relação à marca, mas não para altos níveis de apego em relação à marca (b = -.02 [-.18; .10]). Esses resultados indicam que a menor intenção de aderir à ação de RSC incongruente comparada à ação de RSC congruente para pessoas com baixo nível de apego à marca é provocada pela maior percepção de intenção persuasiva. Por sua vez, pessoas com alto apego à marca são imunes à intenção persuasiva da campanha, razão pela qual não verificamos diferença entre as intenções de aderir em relação às duas campanhas, confirmando $\mathrm{H}_{2}$.

\section{Discussão}

Os resultados do primeiro experimento sugerem que o efeito negativo da incongruência marca-causa sobre a intenção de aderir à ação de RSC só foi significativo para pessoas com baixo apego à marca. Kwak e Kwon (2016) já haviam observado que ações de RSC estimulam os consumidores que se identificam com a organização a também apoiarem a causa, estimulando sentimentos de gratidão que motivam a intenção de também doar/apoiar a ação. Se estendermos os resultados obtidos no primeiro experimento com o apego à marca para a identificação com a organização, nossos resultados sugerem que apenas indivíduos com um alto nível de identificação com a empresa promotora da ação de RSC estarão propensos a também contribuírem para a causa.

Estudos sobre apego à marca verificaram que os indivíduos parecem perceber menos motivações negativas associadas a 
ações de RSC promovidas por marcas às quais são mais apegados (Chiou et al., 2013; Schmalz \& Orth, 2012). Consistentes com esses estudos, os resultados do primeiro experimento sugerem que os indivíduos com baixo apego à marca perceberam níveis mais elevados de intenção persuasiva em ações de RSC incongruentes com a marca, diminuindo sua intenção de adesão. Indivíduos com elevado nível de apego, por outro lado, não reportaram diferenças na percepção de intenção persuasiva e demonstraram a mesma intenção de aderirem à causa de RSC, independentemente da congruência marca-causa.

Embora o apego à marca seja idiossincrático e não esteja sob controle direto dos gerentes de marcas, ele é baseado em sentimentos de segurança construídos durante o relacionamento do indivíduo com a marca (Sen et al., 2015). Tal segurança, fruto da história entre o indivíduo e a marca, parece tornar os consumidores imunes à percepção de motivações oportunistas e, portanto, mais dispostos a apoiarem as ações promovidas pela marca.

\section{EXPERIMENTO 2}

Segundo Drumwright (1996), tão ou até mais importante que a congruência marca-causa é a congruência consumidor-causa pois, quando esta é baixa, o interesse de engajamento do indivíduo é menor. Grau e Folse (2007) mostraram que indivíduos com mais envolvimento demonstraram atitudes mais favoráveis em relação a ações de RSC, e a atitude de indivíduos com menos envolvimento só aumentava quando a ação apoiava causas locais ao invés de causas nacionais. De modo similar, Lichtenstein, Drumwright e Braig (2004) observaram que a relevância da causa para o indivíduo exerce influência sobre a decisão de compra de produtos de marcas associadas à RSC. Zdravkovic et al. (2010) verificaram que a familiaridade do indivíduo com a causa influencia significativamente a atitude em relação à marca quando há congruência marca-causa, mas não quando estas são incongruentes.

Estendendo tais conclusões, Barone et al. (2007) verificaram que a atitude em relação à causa pode também influenciar o efeito da congruência entre a causa e a marca na determinação das respostas dos consumidores. Quando os indivíduos possuem atitudes positivas em relação à causa, a congruência torna-se menos relevante, pois há uma interação entre a afinidade do consumidor com a causa e a percepção dos motivos da empresa promotora da ação. Sheikh e Beise-Zee (2011, p. 27) também investigaram a relação da congruência marcacausa e a afinidade do indivíduo com a causa sobre a atitude em relação à empresa promotora. Sheikh e seu colega observaram que a congruência marca-causa possui efeito significativo sobre a atitude do indivíduo quando a afinidade é baixa, mas o efeito é insignificante quando a afinidade é alta.

Entretanto, os estudos acima investigaram apenas os efeitos da atitude do indivíduo em relação à causa sobre os retornos das ações de RSC para a empresa, e não especificamente para a causa. Kwak e Kwon (2016) observaram que, além da identificação entre consumidor-empresa, a atitude em relação à causa também influencia positivamente a intenção de doação: quanto maior a atitude em relação à causa, mais forte a relação gratidão-doação e, consequentemente, maior a intenção de doação do indivíduo.

Embora os resultados de estudos anteriores sugiram que atitudes mais positivas em relação à causa de RSC conduzam a respostas mais positivas dos consumidores para a marca mesmo quando a congruência marca-causa é baixa, a atitude em relação à causa pode também influenciar a intenção de engajamento do indivíduo (Kwak \& Kwon, 2016). Propomos que atitudes mais favoráveis à causa podem mitigar o efeito de um baixo apego em relação à marca e da baixa congruência marca-causa, ou seja, independentemente do apego à marca e da congruência marca-causa, quando a atitude em relação à causa de RSC é alta, a intenção de aderir à causa de RSC patrocinada por uma marca será alta. Entretanto, quando a atitude em relação à causa não for tão positiva, os resultados observados no primeiro experimento devem se manter, ou seja, o apego em relação à marca moderará o efeito da congruência marca-causa sobre a intenção de aderir à causa. Essa discussão leva às seguintes hipóteses:

$\mathrm{H}_{3 \mathrm{a}}$ : $\mathrm{O}$ efeito previsto em $\mathrm{H}_{1 \mathrm{a}}$ e $\mathrm{H}_{1 \mathrm{~b}}$ se manterá quando o consumidor tiver uma baixa atitude em relação à causa de RSC promovida.

$\mathrm{H}_{3 \mathrm{~b}}$ : A intenção de aderir à ação de RSC não dependerá da congruência marca-causa nem do apego à marca quando $a$ atitude em relação à causa de RSC promovida for alta.

\section{Método}

\section{Amostra e design}

Estudantes universitários ( $n=280,61 \%$ homens, $M=21,5$ anos) foram convidados a participar de um experimento de laboratório 2 (congruência marca-causa: congruente vs. incongruente) X 2 (CocaCola vs. Dove), entre sujeitos, em que o apego à marca e a atitude em relação à causa de RSC foram mensurados. Para aumentar a generalização dos resultados, fizemos duas replicações com duas marcas diferentes (Dove e Coca-Cola) e mantivemos constantes as ações de RSC de tal forma que uma ação de RSC é congruente com uma das marcas e incongruente com a outra. Portanto, esperávamos observar os mesmos resultados para cada uma das 
marcas. Ao contrário de estudos anteriores em que as replicações foram feitas solicitando aos participantes a avaliação de diversas ações de RSC (e.g., Zdravkovic et al., 2010), optamos por expor cada indivíduo a um único estímulo marca-causa para diminuir a carga cognitiva de realizar diversas avaliações. Ao invés de variar as ações para cada marca, preferimos mantê-las constantes, com o objetivo de demonstrar que os resultados observados no primeiro experimento não são resultados de uma escolha adequada marcacausa e se mantêm independentemente da marca.

\section{Procedimentos}

Mantivemos a marca Coca-Cola e a reciclagem coletiva para representar a marca-causa congruente (estímulos usados no primeiro experimento) e, para maior generalização, substituímos a causa "Prevenção contra o câncer de pele" do primeiro experimento por "Prevenção contra o câncer de mama" para representar uma ação incongruente com a marca Coca-Cola. Baseados nos resultados do pré-teste do primeiro experimento, escolhemos a marca Dove por ter sido a mais citada, entre as cinco marcas disponíveis, como mais apropriada para apoiar a ação “Prevenção contra o câncer de mama” (16 citações ou 52\% dos respondentes). Ao fazer essas escolhas, esperávamos que a marca Coca-Cola (Dove) fosse congruente (incongruente) com a ação de reciclagem coletiva e incongruente (congruente) com a causa de prevenção do câncer de mama.

Os estímulos, pretensamente propagandas a serem veiculadas em revistas, foram criados utilizando-se o mesmo layout, design, cores, plano de fundo, fonte e frases semelhantes.

Os participantes responderam às mesmas escalas utilizadas no primeiro experimento adaptadas para este experimento, com exceção da escala de apego à marca. Para tanto, foi utilizada a versão de Park et al. (2010), respondida por meio de uma escala de Likert de 7 pontos ( 1 = discordo totalmente; 7 = concordo totalmente). Além disso, os participantes foram convidados a avaliar a sua atitude em relação à causa de RSC usando os cinco itens da escala de Webb, Green e Brashear (2000). Depois de completar essas medidas, os participantes responderam algumas questões demográficas e receberam um agradecimento pela participação.

\section{Resultados}

\section{Conferência das manipulações}

Após o agrupamento das questões relacionadas à congruência marca-causa $(M=4,74 ; D P=1,66 ; a=.89)$, verificamos que $a$ congruência percebida entre a marca Coca-Cola e a campanha de reciclagem coletiva $(M=5,6)$ foi maior do que a congruência entre a marca Coca-Cola e a causa de combate ao câncer de mama $(M=3.5 ; t(138)=-8,82 ; p<0,01)$. Da mesma forma, a congruência percebida entre a marca Dove e a causa de combate ao câncer de mama foi maior $(M=5,5)$ do que a congruência entre a marca Dove e a causa de reciclagem coletiva $(M=4,4 ; t(138)=-8,66$; p<0,01). Portanto, a manipulação da congruência entre as ações e as marcas funcionou.

\section{Intenção de aderir à campanha}

Para verificar se os resultados foram os mesmos para cada uma das duas replicações, fizemos uma análise de regressão usando como variável dependente a intenção de aderir à causa e como independentes as variáveis dummy correspondentes às marcas e às causas de RSC, o apego em relação à marca, a atitude em relação à causa e todas as interações entre as quatro variáveis. A regressão revelou-se significativa $\left(F(15,264)=6,81(p<0,01) ; R^{2}=.24\right)$, mas nem o coeficiente associado à dummy correspondente às marcas nem nenhuma das interações com essa dummy foram significativas (todos os p's > 0,30). Isso significa que, assim como esperávamos, a marca não interagiu com nenhuma das variáveis e, portanto, para o teste das hipóteses, poderíamos agrupar os dados das duas marcas.

Para o teste das hipóteses $\mathrm{H}_{3 \mathrm{a}}$ e $\mathrm{H}_{3 \mathrm{~b}}$, utilizamos a macro PROCESS (Hayes, 2013; Modelo 3). 0 procedimento foi feito usando a variável intenção de aderir como dependente, o apego à marca $\mathrm{e}$ a atitude em relação à causa como moderadores e a congruência da campanha ( 0 = incongruente; 1 = congruente) como variável independente. Como esperávamos, os resultados revelaram que 0 coeficiente do termo associado à interação entre as três variáveis foi significativo $(b=0,21 ; t(272)=2,16, p<0,05)$. Para a amostra de 280 respondentes, o cálculo post-hoc do poder do teste do teste $t$ do coeficiente de interação, calculado pelo software G-Power (Faul et al., 2007), foi constatado superior a 99,9\%.

Para decompor a interação entre as três variáveis e facilitar a interpretação de acordo com as hipóteses, foram feitas duas análises separadas, uma para níveis baixos de atitude em relação à causa de $\mathrm{RSC}\left(\mathrm{H}_{3 \mathrm{a}}\right)$ e outra para níveis altos de atitude em relação à causa $\left(\mathrm{H}_{3 \mathrm{~b}}\right)$. Uma vez que a variável atitude em relação à causa foi mensurada, os dois grupos foram divididos pela mediana (Med $=5,8)$. Também fizemos a análise usando o mesmo procedimento com todos os valores da escala de atitude em relação à causa, e os resultados foram os mesmos que aqueles obtidos pela dicotomização. Para facilitar a compreensão dos resultados, entretanto, preferimos apresentar apenas o resultado obtido com a dicotomização da variável atitude em relação à causa. 
Para níveis baixos de atitude em relação à causa, uma análise similar àquela usada no primeiro experimento (PROCESS, Modelo 1) revelou uma interação significativa entre apego à marca e congruência marca-causa $(b=-0,34 ; t(139)=-2.26, p<0,05)$. A técnica Johnson-Neyman revelou um efeito positivo e significativo da congruência $(b=0,40, p=0,05)$ para níveis de apego inferiores a 2,60 , representando $66 \%$ da amostra. Em outras palavras, quando a atitude em relação à causa é baixa, a causa congruente gera maior intenção de adesão do que a causa incongruente para baixos níveis de apego (inferior a 2,60), mas não para altos níveis de apego, confirmando $\mathrm{H}_{3 \mathrm{a}}$ (Gráfico 2, Painel $A$ )

A mesma análise foi feita para níveis altos de atitude em relação à causa. Os resultados revelaram que o coeficiente associado à interação congruência marca-causa e ao apego à marca não foi significativo $(b=-0,11 ; \mathrm{t}(133)=-0,85, \mathrm{p}=0,40)$; 0 apego em relação à marca $(b=0,19 ; \mathrm{t}(133)=1,98, \mathrm{p}<0,05)$ foi significativo e o coeficiente associado à ação de RSC ficou próximo da significância $(b=.66, \mathrm{t}(133)=1.65 ; \mathrm{p}=0,1003)$. Portanto, os resultados sugerem que, para altos níveis de atitude em relação à causa, o apego em relação à marca não modera o efeito da congruência marca-causa sobre a intenção de aderir à causa, confirmando $\mathrm{H}_{3 \mathrm{~b}}$ (Gráfico 2, Painel B). Por outro lado, os resultados também sugerem que a intenção de aderir à causa aumenta com o apego à marca e, de modo direcional, é maior para a causa congruente com a marca comparada à causa incongruente.

\section{Gráfico 2. Intenção de aderir à causa por congruência e por nível de apego à marca}

Painel A

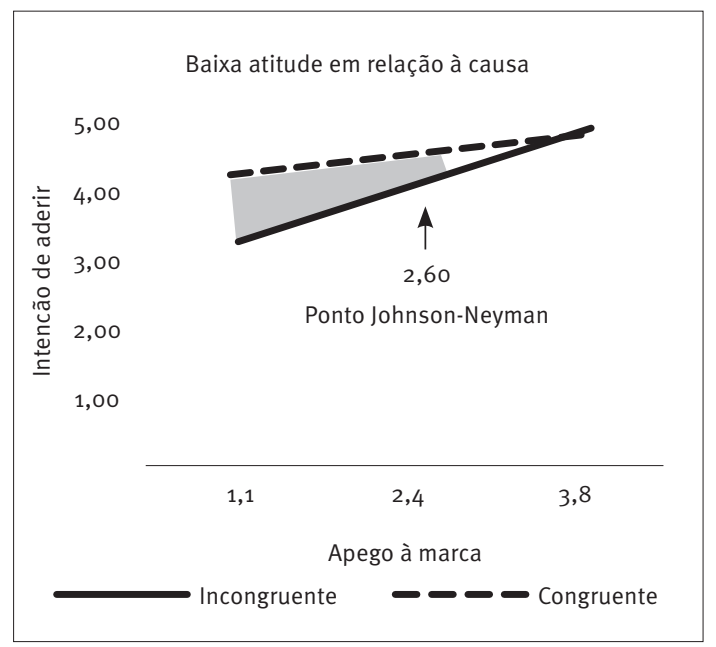

Painel B

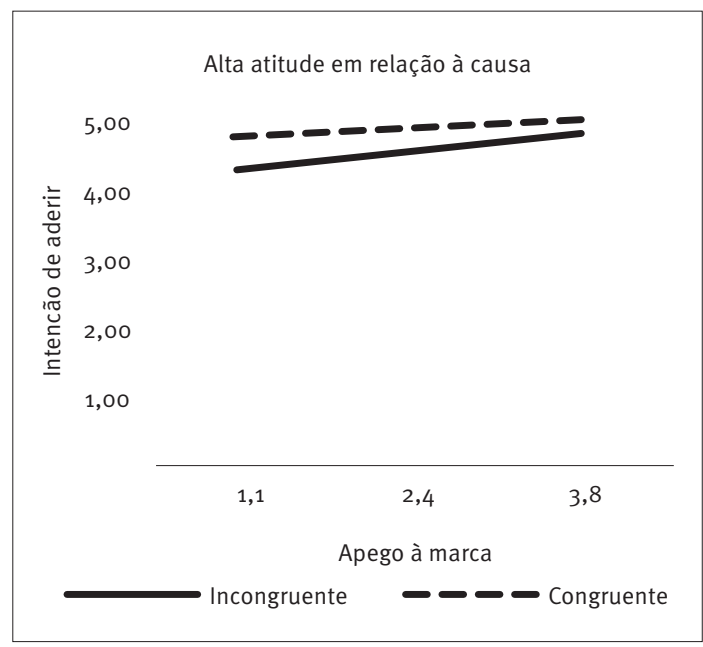

\section{Discussão}

$\mathrm{O}$ estudo 2 fornece suporte para as hipóteses $\mathrm{H}_{3 \mathrm{a}}$ e $\mathrm{H}_{3 b}$. Esse resultado é compatível com os resultados dos estudos de Barone et al. (2007) e Kwak e Kwon (2016). Para situações de alta atitude em relação à causa, a congruência marca-causa não influenciou as respostas dos indivíduos em relação ao apoio à causa, ainda que a intenção de aderir à causa esteja relacionada ao apego à marca. No entanto, se a atitude é baixa, o apego do indivíduo à marca será um importante motivador ao apoio dessas ações. Já se o apego e a atitude forem baixos, a congruência marca-causa torna-se o elemento definidor da intenção de adesão à causa. Além disso, para níveis baixos de atitude em relação à causa, os resultados do segundo experimento replicam os resultados do primeiro experimento, mas com duas marcas diferentes e causas diferentes, aumentando a capacidade de generalização dos resultados.

\section{DISCUSSÃO GERAL}

Por meio dos resultados de dois experimentos, verificamos que o patrocínio de ações de RSC por uma marca influencia positivamente a intenção dos indivíduos em apoiar tais ações. Entretanto, como previmos, a maior ou menor intenção dos consumidores de aderir às causas apoiadas pelas marcas é 
contingente à congruência marca-causa, ao apego em relação à marca e à atitude em relação à causa.

Usando diferentes marcas e causas como estímulo, verificamos que, quando a atitude dos consumidores em relação à causa é baixa, a intenção de aderir à causa é maior quando a congruência marca-causa é maior, mas apenas quando o apego à marca é baixo; quando o apego é alto, a congruência marcacausa torna-se irrelevante para o apoio do consumidor. Por outro lado, quando a atitude do consumidor em relação à causa é alta, a intenção de aderir à causa aumenta à medida que aumenta o apego. Além disso, verificamos que a percepção de intenção persuasiva diminui a intenção de aderir à causa quando a congruência é baixa para consumidores com baixo apego à marca.

Os resultados deste estudo têm importantes contribuições teóricas e gerenciais. Em primeiro lugar, propusemos um quadro teórico que investiga o efeito de três variáveis que não haviam sido examinadas de maneira conjunta anteriormente: a congruência marca-causa, o apego à marca e a atitude em relação à causa. A integração de variáveis que se mostraram relevantes quando analisadas isoladamente em um único modelo teórico aumenta tanto a capacidade de previsão quanto a de explicação de um fenômeno de interesse, como o patrocínio de ações de RSC. Apenas ao integrar essas três variáveis é que foi possível identificar que a atitude em relação à causa inibe os efeitos negativos de um nível baixo de apego à marca e de uma baixa congruência marca-causa.

A segunda contribuição deste estudo foi mostrar que ações de RSC com uma marca podem ser percebidas como incoerentes ou inapropriadas por consumidores de baixo apego à marca, aumentando a percepção de intenção persuasiva da ação e reduzindo a intenção de aderir à causa. Embora estudos anteriores (Romani \& Grappi, 2014; Sharkmeas \& Leonidou, 2013; Vlachos et al., 2009) houvessem sugerido teoricamente essa relação, nenhum estudo havia testado esse efeito mediador empiricamente. Além do mais, os resultados relatados aqui confirmam que o apego à marca funciona como uma barreira protetora à reputação das marcas (Chiou et al., 2013; Fedorikhin, Park, \& Thomson, 2008; Schmalz \& Orth, 2012), uma vez que indivíduos que têm alto apego à marca não perceberam a intenção persuasiva de ações de RSC incongruentes com a marca.

A terceira contribuição do estudo está na investigação da intenção dos consumidores de também aderirem à causa promovida por uma marca. Enquanto os benefícios do patrocínio de ações de RSC para as marcas estão bem-documentados (Becker-Olsen et al., 2006; Mohr et al., 2001), os resultados sociais dessas ações têm recebido muito menos atenção em estudos anteriores da área (para exceções, veja Lichtenstein et al.,
2004; Romani \& Grappi, 2014; Romani et al., 2014). À medida que os problemas sociais se tornam mais complexos, a participação de um número maior de pessoas em ações de RSC lideradas por marcas torna-se mais relevante. Por exemplo, em sua campanha para ajudar a fornecer água potável para as pessoas que não têm acesso a esse item essencial, a Stella Artois e sua parceira Water. Org já venderam mais de 225 mil cálices de vidro que tiveram o valor total arrecado (mais de 3 milhões de dólares) doado para a causa (Stella Artois, 2017). Da mesma forma, a campanha McDia Feliz, promovida há 29 anos pelo McDonald's, arrecadou mais de 25 milhões de reais em 2017, valor destinado ao tratamento de crianças com câncer (McDia Feliz, 2017). Resultados como esses só foram possíveis porque os consumidores se engajaram numa campanha social liderada por uma marca.

Da mesma forma, o presente estudo tem importantes contribuições gerenciais ao proporcionar direções estratégicas aos gerentes de marcas que pretendem engajar seus consumidores em ações de RSC. Ainda que os resultados tenham mostrado que a atitude em relação à causa seja um importante preditor do engajamento dos consumidores em ações de RSC promovidos pelas marcas e possa reduzir o efeito negativo do baixo apego à marca e da baixa congruência marca-causa, gerentes de marcas devem ser cuidadosos na escolha das ações de RSC a serem patrocinadas. Os resultados mostram que o engajamento dos consumidores em ações incongruentes tende a ser menor, principalmente para as pessoas que têm baixo apego à marca.

Por outro lado, a promoção de ações de RSC também pode fortalecer as relações entre os consumidores e as marcas, tendo reflexos positivos na construção do apego à marca (Bhattacharya \& Sen, 2004; Stanaland, Lwin, \& Murphy, 2011). Quando os consumidores se engajam nas ações de RSC, as pessoas percebem que o esforço da marca é justo e sentem-se parceiras da marca na solução dos problemas sociais. Essa ação conjunta tem resultados positivos não apenas para as pessoas beneficiadas pela ação social, mas também pode resultar em benefícios para as marcas.

Park et al. (2010) sugeriram que quanto mais forte 0 apego do indivíduo à marca, maior a disposição a se engajar em comportamentos que exijam investimentos de tempo, dinheiro, energia ou reputação para fortalecer ou manter o relacionamento com a marca-alvo do apego. 0 apego aumenta a empatia e a identificação do indivíduo com a empresa, além de motivar comportamentos de comprometimento (Hazan \& Shaver, 1994). Portanto, o patrocínio de ações socialmente responsáveis parece ter inspirado nos respondentes deste estudo o desejo de demonstrar comportamentos exemplares e pró-sociais.

Por fim, os resultados também indicam que o engajamento nas ações de RSC patrocinadas pelas marcas é maior entre 
pessoas com alto apego à marca e atitudes mais positivas em relação à causa. Portanto, as marcas podem obter resultados melhores e mais rápidos ao direcionarem seus esforços de patrocínio de ações de RSC a segmentos de consumidores que possuam essas características.

\section{Limitações e estudos futuros}

Nos experimentos relatados aqui, tanto o apego quanto a atitude em relação à causa foram medidos e não manipulados. A manipulação do apego é uma tarefa difícil de ser executada em condições experimentais, pois o apego é construído e fortalecido em decorrência do tempo (Baldwin, Keelan, Fehr, Enns, \& KohRangarajoo, 1996). A manipulação do apego nos daria maiores garantias de que esse constructo não está confundido com variáveis similares, como a atitude em relação à marca. Entretanto, como a atitude não possui o mesmo grau de intensidade que o apego, provavelmente não observaríamos os mesmos efeitos. Por outro lado, a atitude em relação à causa pode ser manipulada com mais facilidade, podendo ser objeto de estudo no futuro.

Os presentes resultados foram obtidos em experimentos realizados com alunos de graduação, portanto estudos futuros podem investigar esses efeitos em populações mais amplas. Além disso, foram investigadas apenas duas marcas, e estudos futuros podem contemplar um número maior de marcas e causas. Da mesma forma, cabe investigar se os efeitos obtidos para a intenção de aderir às causas se manteriam para variáveis de interesse das marcas, como a intenção de aumentar o consumo da marca ou a atitude em relação à marca.

\section{REFERÊNCIAS}

Andrews, M., Luo, X., Fang, Z., \& Aspara, J. (2014). Cause marketing effectiveness and the moderating role of price discounts. Journal of Marketing, 78(6), 120-142. doi:10.1509/jm.14.0003

Baldwin, M., Keelan, J., Fehr, B., Enns, V., \& Koh-Rangarajoo, E. (1996). Social-cognitive conceptualization of attachment working models: Availability and accessibility effects. Journal of Personality and Social Psychology, 71(1), 94-109. doi:10.1037/0022-3514.71.1.94

Barone, M. J., Norman, A. T., \& Miyazaki, A. D. (2007). Consumer response to retailer use of cause-related marketing: Is more fit better? Journal of Retailing, 83(4), 437-445. doi:10.1016/j.jretai.2007.03.006

Becker-Olsen, K. L., Cudmore, B. A., \& Hill, R. P. (2006). The impact of perceived corporate social responsibility on consumer behavior. Journal of Business Research, 59(1), 46-53. doi:10.1016/j. jbusres.2005.01.001
Bhattacharya, C. B., \& Sen, S. (2004). Doing better at doing good: When, why, and how consumers respond to corporate social initiatives. California Management Review, 47(1), 9-24. doi:10.2307/41166284

Campbell, M. C. (1995). When attention-getting advertising tactics elicit consumer inferences of manipulative intent: The importance of balancing benefits and investments. Journal of Consumer Psychology, 4(3), 225-254. doi:10.1207/s15327663jcpo403_02

Chiou, J.-S., Hsu. A. C-F., Hsieh, C-H. (2013). How negative online information affects consumers' brand evaluation: The moderating effects of brand attachment and source credibility. Online Information Review, 37(6), 910-926. doi:10.1108/0IR-02-2012-0014

Drumwright, M. E. (1996). Company advertising with a social dimension: The role of noneconomic criteria. Journal of Marketing, 60(4), 71-87. doi:10.2307/1251902

Faul, F., Erdfelder, E., Lang, A.-G., \& Buchner, A. (2007). G*Power 3: A flexible statistical power analysis program for the social, behavioral, and biomedical sciences. Behavior Research Methods, 39(2), 175-191. doi:10.3758/BFo3193146

Fedorikhin, A., Park, C. W., \& Thomson, M. (2008). Beyond fit and attitude: The effect of emotional attachment on consumer responses to brand extensions. Journal of Consumer Psychology, 18(4), 281-291. doi:10.1016/j.jcps.2008.09.006

Friestad, M., \& Wright, P. (1994). The persuasion knowledge model: How people cope with persuasion attempts. Journal of Consumer Research, 21(1), 1-31.

Grau, S. L., \& Folse, J. A. G. (2007). Cause-related marketing (CRM): The influence of donation proximity and message-framing cues on the less-involved consumer. Journal of Advertising, 36(4), 19-33.

Hayes, A. F. (2013). Introduction to mediation, moderation, and conditional process analysis: A regression-based approach. New York, NY: Guilford Press.

Hazan, C., \& Shaver, P. R. (1994). Attachment as an organizational framework for research on close relationships. Psychological Inquiry, 5(1), 1-22. doi:10.1207/s15327965plio501_1

He, H., Zhu, W., Gouran, D., \& Kolo, O. (2016). Moral identity centrality and cause-related marketing: The moderating effects of brand social responsibility image and emotional brand attachment. European Journal of Marketing, 50(1/2), 236-259. doi:10.1108/EJM-10-20140613

Keller, K. L., \& Aaker, D. A. (1992). The effects of sequential introduction of brand extensions. Journal of Marketing Research, 29(1), 35-50.

Kwak, D. H., \& Kwon, Y. (2016). Can an organization's philanthropic donations encourage consumers to give? The roles of gratitude and boundary conditions. Journal of Consumer Behaviour, 15(4), 348-358. doi:10.1002/cb.1576

Lawler, E. J., \& Thye, S. R. (2006). Social exchange theory of emotions. In J. E. Stests \& J. H. Turner (Eds.), Handbook of the sociology of emotions (pp. 295-320). New York, USA: Springer.

Lichtenstein, D. R., Drumwright, M. E., \& Braig, B. M. (2004). The effect of corporate social responsibility on customer donations to corporatesupported nonprofit. Journal of Marketing, 68(4), 16-32.

Lord, C. G., Ross, L., \& Lepper, M. R. (1979). Biased assimilation and attitude polarization: The effects of prior theories on subsequently considered evidence. Journal of Personality and Social Psychology, 37(11), 2098-2109. doi:10.1037/0022-3514.37.11.2098 
Maignan, I., \& Ferrell, O. C. (2004). Corporate social responsibility and marketing: An integrative framework. Journal of the Academy of Marketing Science, 32(1), 3-19. doi:10.1177/0092070303258971

McCullough, M. E., Rachal, K. C., Sandage, S. J., Worthington, E. L., Jr,. Brown, S. W., \& Hight, T. L. (1998). Interpersonal forgiving in close relationships: II. Theoretical elaboration and measurement. Journal of Personality and Social Psychology, 75(6), 1586-1603. doi:10.1037/0022-3514.75.6.1586

McDia Feliz. (2017). O McDia Feliz 2017 foi um sucesso. Recuperado de https://mcdiafeliz.org.br/noticias/o-mcdia-feliz-2017-foi-um-sucesso/

Mohr, L., Webb, D., \& Harris, K. (2001). Do consumers expect companies to be socially responsible? The impact of corporate social responsibility on buying behavior. Journal of Consumer Affairs, 35(1), 45-72. doi:10.1111/j.1745-6606.2001.tbo0102.x

Nan, X., \& Heo, K. (2007). Consumer responses to corporate social responsibility (CSR) initiatives: Examining the role of brand-cause fit in cause-related marketing. Journal of Advertising, 36(2), 63-74.

Obermiller, C., \& Spangenberg, E. R. (1998). Development of a scale to measure consumer skepticism toward advertising. Journal of Consumer Psychology, 7(2), 159-186. doi:10.1207/ S15327663jicpo702_03

Park, C. W., MacInnis, D. J., \& Priester, J. (2008). Brand attachment: Constructs, consequences, and causes. Foundations and Trends $₫$ in Marketing, 1(3), 191-230. doi:10.1561/1700000006

Park, C. W., Maclnnis, D. J., Priester, J., Eisingerich, A. B., \& lacobucci, D. (2010). Brand attachment and brand attitude strength: Conceptual and empirical differentiation of two critical brand equity drivers. Journal of Marketing, 74(6), 1-17. doi:10.1509/jmkg.74.6.1

Romani, S., \& Grappi, S. (2014). How companies' good deeds encourage consumers to adopt pro-social behavior. European Journal of Marketing, 48(5/6), 943-963. doi:10.1108/EJM-06-2012-0364

Romani, S., Grappi, S., \& Bagozzi, R. P. (2014). Corporate socially responsible initiatives and their effects on consumption of green products. Journal of Business Ethics, 135(2), 253-264. doi:10.1007/ s10551-014-2485-0

Roy, D. P. (2010). The impact of congruence in cause marketing campaigns for service firms. Journal of Services Marketing, 24(3), 255-263. doi:10.1108/08876041011040659

Schmalz, S., \& Orth, U. R. (2012). Brand attachment and consumer emotional response to unethical firm behavior. Psychology \& Marketing, 29(11), 869-884. doi:10.1002/mar.20570

Sen, S., \& Bhattacharya, C. B. (2001). Does doing good always lead to doing better? Consumer reactions to corporate social responsibility. Journal of Marketing Research, 38(2), 225-243. doi:10.1509/ jmkr.38.2.225.18838
Sen, S., Bhattacharya, C. B., \& Korschun, D. (2006). The role of corporate social responsibility in strengthening multiple stakeholder relationships: A field experiment. Journal of the Academy of Marketing Science, 34(2), 158-166.

Sen, S., Johnson, A. R., Bhattacharya, C. B., \& Wang, J. (2015). Identification and attachment in consumer-brand relationships. In N. K. Malhotra, D. Maclnnis, \& C. W. Park (Eds.), Brand meaning management (Review of marketing research) (Vol. 12, pp. 151-174). Bingley, UK: Emerald Group Publishing

Sheikh, S., \& Beise-Zee, R. (2011). Corporate social responsibility or causerelated marketing? The role of cause specificity of CSR. Journal of Consumer Marketing, 28(1), 27-39. doi:10.1108/07363761111101921

Skarmeas, D., \& Leonidou, C. N. (2013). When consumers doubt, watch out! The role of CSR skepticism. Journal of Business Research, 66(10), 1831-1838. doi:10.1016/j.jbusres.2013.02.004

Smith, D. C., \& Park, C. W. (1992). The effects of brand extensions on market share and advertising efficiency. Journal of Marketing Research, 29(3), 296-313. doi:10.2307/3172741

Spiller, S. A., Fitzsimons, G. J., Lynch, J. G., \& McClelland, G. H. (2013). Spotlights, floodlights, and the magic number zero: Simple effects tests in moderated regression. Journal of Marketing Research, 50(2), 277-288. doi:10.1509/jmr.12.0420

Stanaland, A. J. S., Lwin, M. O., \& Murphy, P. E. (2011). Consumer perceptions of the antecedents and consequences of corporate social responsibility. Journal of Business Ethics, 102(1), 47-55. doi:10.1007/s10551-011-0904-z

Stanwick, P. A., \& Stanwick, S. D. (1998). The relationship between corporate social performance and organizational size, financial performance, and environmental performance: An empirical examination. Journal of Business Ethics, 17(2), 195-204.

Stella Artois. (2017). Buy a lady a drink. Recuperado de https://www. stellaartois.com.br/1-calice-5-anos

Thomson, M., MacInnis, D. J., \& Park, C. W. (2005). The ties that bind: Measuring the strength of consumers' emotional attachments to brands. Journal of Consumer Psychology, 15(1), 77-91. doi:10.1207/ S15327663jcp1501_10

Vlachos, P. A., Tsamakos, A., Vrechopoulos, A. P., \& Avramidis, P. K. (2009). Corporate social responsibility: Attributions, loyalty, and the mediating role of trust. Journal of the Academy of Marketing Science, 37(2), 170-180. doi:10.1007/s11747-008-0117-x

Webb, D. J., Green, C. L., \& Brashear, T. G. (2000). Development and validation of scales to measure attitudes influencing monetary donations to charitable organizations. Journal of the Academy of Marketing Science, 28(2), 299-309. doi:10.1177/0092070300282010

Zdravkovic, S., Magnusson, P., \& Stanley, S. M. (2010). Dimensions of fit between a brand and a social cause and their influence on attitudes. International Journal of Research in Marketing, 27(2), 151160. doi:10.1016/j.ijresmar.2010.01.005 